\title{
A Moving Mesh Algorithm for Aero-Thermo-Mechanical Modeling in Turbomachinery
}

\author{
D. Amirante ${ }^{1 *}$, N.J. Hills ${ }^{1}$ and C.J. Barnes ${ }^{2}$ \\ ${ }^{1}$ University of Surrey, Guildford, GU2 7TZ, UK \\ ${ }^{2}$ Rolls Royce plc, Derby, GU2 7TZ, UK
}

\begin{abstract}
SUMMARY
This paper describes the development of a mesh deformation method used for aero-thermo-mechanical coupling of turbo-engine components. The method is based on the nonlinear solution of an elastic medium analogy, solved using finite element discretization, and modified to let the boundary nodes be free to slide over the deflected surfaces. This sliding technique relies on a B-Spline reconstruction of the moving boundary, and increases the robustness of the method in situations where the boundary deflection field presents significant gradients, or large relative motion between two distinct boundaries. The performance of the method is illustrated with the application to an interstage cavity of a turbine assembly, subjected to the deformations computed by a coupled thermo-mechanical analysis of the engine component. Copyright (c) 0000 John Wiley \& Sons, Ltd.
\end{abstract}

Received ...

KEY WORDS: Fluid-structure interaction; Mesh generation; Shape design.

\section{INTRODUCTION}

The prediction of metal temperatures plays an essential role in the design of modern gas turbines. The optimization of heat transfer between fluid and metal can help to minimize the coolant flow requirements, with a direct reduction of the corresponding loss in the thermodynamic cycle. With the increase of computing power, coupling techniques are rapidly becoming a common tool for solid/fluid heat transfer modeling. In these methods, Computational Fluid Dynamics (CFD) is used to predict the heat flux from the air to the metal, and this is coupled to the thermal analysis predicting metal temperatures. Much of this work has been applied to turbine blade cooling applications, with early work, for example, by Heselhaus et al. [1], Chew et al. [2], and the more recent studies by He and Oldfield [3]. In recent years this has been extended to disc cavity flow, for example by Verdicchio et al. [4] and Sun et al. [5].

In most of these studies, the coupling between the solid and fluid domain is limited to the temperature and heat flux, that is, the analysis is conducted on a given cold geometry. However, this

\footnotetext{
*Correspondence to: d.amirante@ surrey.ac.uk

Copyright (c) 0000 John Wiley \& Sons, Ltd.

Prepared using nmeauth.cls [Version: 2010/05/13 v3.00]
} 
may be inadequate in situations where the metal deformation affects the developing flow dynamics. For example, in rotor-stator cavities, the thermo-mechanical distortions govern the labyrinth seal clearance. This is directly related to the amount of hot air ingested from the main annulus, which in turn determines the resulting heat flux. Such a feedback loop can be taken into account by integrating the fluid-structure interaction in the coupling process, so that the CFD is always being carried out using the hot running geometry. In this context, a critical issue is the capability to modify an existing mesh, or to create a new one, in order to conform with the evolving geometry.

A dynamic mesh is generally referred to as a mesh able to deform while preserving its original nodal connectivity. This feature renders the use of dynamic meshes more attractive than the alternative option of re-meshing. In the latter case, difficulties related to the projection of the solution from the old mesh to the new one can occur, and, most importantly, manual intervention may be necessary. Clearly, this represents a major obstacle if the final goal is to achieve full automation of multidisciplinary interactions. These considerations explain the large and enduring interest that dynamic meshes generate.

The most common techniques assign structural properties to the original mesh, and determine the nodal displacements as the solution of an equilibrium problem. For the classic edge/torsional spring analogy methods $[6,7]$ the initial mesh is directly seen as a discrete structure, while in the elastic medium analogy [8] the domain is considered as a continuous elastic medium which is subsequently discretized. From the perspective of their numerical interpretation, however, this difference is little more than formal. In either case, one ends up with a finite element discretization of a structure represented by a stiffness matrix $\mathbf{K}$, and many studies on dynamic meshes have attempted to provide the most appropriate way to define $\mathbf{K}$. These include the major contributions of Farhat et al. [7] and Degand \& Farhat [9], the ortho-semi-torsional spring method of Markou et al. [10], the ball vertex method of Bottasso et el. [11], and the semi-torsional approach of Zeng \& Ethier [12] for the category of spring analogy, while in the context of the elastic medium analogy we mention the works of Johnson and Tezduyar [8, 13], Stein et al. [14], and the more recent method proposed by Hsu and Chang [15] based on the Tresca failure criterion. The aim of these studies is generally the same: enhancing the robustness of the method with respect to boundary deflections, topological complexity and mesh refinement.

This paper details a moving mesh method for aero-thermo-mechanical coupling used in turbomachinery design. This kind of application belongs to the class of problems for which the robustness of the method is more important than its efficiency. In fact, in the thermal coupling the mesh is deformed with a frequency dictated by the time scale of the metal heat conduction. This circumstance allows the employment of methods which are known to be computationally expensive, but robust enough to handle the additional difficulties arising from the geometric complexity. The dynamic mesh is computed using an elastic medium analogy with geometric nonlinearity. The method is supplemented with a sliding technique, integrating the computation for the displacement of boundary nodes into the elastic problem. Given a stiffness matrix, the equation solved on the boundary nodes is modified so as to let them free to move over the deflected surfaces. Despite the simplicity of the underlying concept, to our knowledge the strategy has not been applied before. This sliding technique, combined with projection operations necessary to preserve geometric consistency, has proved to be critical for the success of the application described. 
The paper is organised as follows: first, the computational method and the details of the numerical implementation are described. This will serve to formalize the introduction of the sliding technique, and to highlight the extra-coding required to extend an existing solver for structural analogies. A 2D model problem is then described before the full application to a turbomachinery test case. The construction of the stiffness matrix follows standard procedures [8,14], and is presented in an appendix for completeness.

The method has been coded following a parallel unstructured implementation, and is valid for both structured and unstructured meshes. However, the examples presented in the paper refer to structured meshes only, since, in general, the turbomachinery community prefers to work with structured meshes.

\section{STRUCTURAL ANALOGY}

We consider a three-dimensional structured mesh subjected to prescribed boundary displacements $\mathbf{u}_{\mathbf{B}}$, and denote by $H$ a generic hexahedral element. The basic idea behind spring or elastic medium analogy methods is to associate fictitious structural properties to the mesh, and to compute the displacements of interior nodes from the deformation of the structure driven by the boundary displacements $\mathbf{u}_{\mathrm{B}}$. In the most straightforward approach, the elasticity problem can be formulated at the discrete level, requiring the balance of the forces produced on the grid node $i$ by all the surrounding elements. Formally, this is written as

$$
\mathbf{F}_{i}=\sum_{H \supset i} \mathbf{F}_{i}^{H}=0
$$

with $\mathbf{F}_{i}^{H}$ representing the nodal force produced by the element $H$ on the node $i$. The sets of forces

and displacements associated with an hexahedral element $H$ are denoted by $\left\{\mathbf{u}^{H}\right\}$ and $\left\{\mathbf{F}^{H}\right\}$, and defined as follows:

$$
\left\{\mathbf{F}^{H}\right\}=\left\{\begin{array}{c}
\mathbf{F}_{1}^{H} \\
\mathbf{F}_{2}^{H} \\
\cdots \\
\cdots \\
\mathbf{F}_{8}^{H}
\end{array}\right\} \quad ; \quad\left\{\mathbf{u}^{H}\right\}=\left\{\begin{array}{c}
\mathbf{u}_{1}^{H} \\
\mathbf{u}_{2}^{H} \\
\cdots \\
\ldots \\
\mathbf{u}_{8}^{H}
\end{array}\right\}
$$

In the finite element approach, the nodal force $\mathbf{F}_{i}^{H}$ is expressed as a linear combination of the displacements associated with the element $H$ :

$$
\mathbf{F}_{i}^{H}=\left[\mathbf{B}_{i}^{H}\right]\left[\mathbf{K}^{H}\right]\left\{\mathbf{u}^{H}\right\}
$$

where $\left[\mathbf{K}^{H}\right]$ is a $24 \times 24$ local stiffness matrix, characterizing the structural properties of the element, and $\left[\mathbf{B}_{i}^{H}\right]$ is a Boolean $3 \times 24$ matrix, defined equivalently by one of the following relations:

$$
\mathbf{F}_{i}^{H}=\left[\mathbf{B}_{i}^{H}\right]\left\{\mathbf{F}^{H}\right\} \quad ; \quad \mathbf{u}_{i}=\left[\mathbf{B}_{i}^{H}\right]\left\{\mathbf{u}^{H}\right\} .
$$


In other words, $\left[\mathbf{B}_{i}^{H}\right]$ is a topological matrix. If $i \in H,\left[\mathbf{B}_{i}^{H}\right]$ extracts from the sets of $\left\{\mathbf{F}^{H}\right\}$ and $\left\{\mathbf{u}^{H}\right\}$, the force $\mathbf{F}_{i}^{H}$ and displacement $\mathbf{u}_{i}$ pertaining to the node $i$. Conversely, if $i \notin H,\left[\mathbf{B}_{i}^{H}\right]$ has all zero entries. The substitution of eq. (3) into (1) yields

$$
\sum_{H \supset i}\left[\mathbf{B}_{i}^{H}\right]\left[\mathbf{K}^{H}\right]\left\{\mathbf{u}^{H}\right\}=0
$$

corresponding to three homogeneous scalar equations whose coefficients are non zero only for the terms involving nodes of the mesh belonging to the same elements as $i$. For boundary points, the equation (5) has to be replaced by the identity specifying the prescribed boundary deflection, that is:

$$
\mathbf{u}=\mathbf{u}_{\mathbf{B}}
$$

For an interior node sharing the same element as a boundary point, the identity (6) provides the non-homogeneous part for the corresponding equations (5). The final linear system of equations $\mathbf{K u}=\mathbf{f}$, involves only the internal points, with the forcing term on the right hand side deriving from the conversion of a Dirichlet-type problem (imposed displacements) into a Neumann-type problem (imposed tractions).

Since the coefficients of the stiffness matrix depend on the geometry only, an important refinement of the method is to assume geometric nonlinearity for the pseudo-structural problem introduced. The global equilibrium $\mathbf{K u}=\mathbf{f}$ can be interpreted as the residual of the nonlinear equation:

$$
\mathbf{R}(\mathbf{u})=\mathbf{K}(\mathbf{u}) \mathbf{u}-\mathbf{f} .
$$

A Newton-Raphson method can be employed, solving at each iteration the system

$$
\mathbf{K}^{(n)} \mathbf{p}=-\mathbf{R}^{(n)},
$$

in which $\mathbf{p}$ is the difference between two consecutive solutions, $\mathbf{p}=\mathbf{u}^{(n+1)}-\mathbf{u}^{(n)}$, whereas $\mathbf{K}^{(n)}$ and $\mathbf{R}^{(n)}$ are the values of the stiffness matrix and the residual corresponding to the solution $\mathbf{u}^{(n)}$.

The drawback of this approach is clearly the computational cost: if one has to deal with large deformations obtained through many sufficiently small mesh motions (typical of aeroelastic applications), nonlinearity introduces unnecessary computations. However, the method has an attractive implication. The Newton-Raphson cycle corresponds to enforcing the global equilibrium $\mathbf{K} \mathbf{u}=\mathbf{f}$ on a structure $\mathbf{K}$ iteratively updated according to the nodal displacements computed at the previous iteration. Suppose that for some mesh nodes we are interested in preserving some geometric requirements, such as, for example, being constrained to remain attached to a specific surface. The nodal update, based on the elastic problem, may not necessarily guarantee the geometric constraints, so that a further correction will be required. A converged solution for the nonlinear elastic problem, provided that it exists, will satisfy both the equilibrium and the requested geometric conditions. This latter property represents, in essence, the basis for the sliding technique introduced in section $\S 4$. 


\section{SOLUTION ALGORITHM}

When dealing with meshes with a large number of points, it can be necessary to employ iterative solvers not requiring the assembly of the global stiffness matrix. The preconditioned conjugate gradient method (PCG) has been adopted in its matrix-free formulation. Before invoking the linear

solver, the program computes and stores the local stiffness matrices $\left[\mathbf{K}^{H}\right]$ (whose construction is given in the appendix), and then calculates the residual $\mathbf{R}$ on the right hand side of the final system to solve (8). The entry component to $\mathbf{R}$ from each internal node $i$ corresponds to the nodal force, and is given by:

$$
\{\mathbf{R}\}_{i}=\sum_{H \supset i} \mathbf{F}_{i}^{H}=\sum_{H \supset i}\left[\mathbf{B}_{i}^{H}\right]\left[\mathbf{K}^{H}\right]\left\{\mathbf{u}^{H}\right\}
$$

The residual is computed with a loop over the set of elements, by adding up the contribution to $\{\mathbf{R}\}_{i}$ from each element sharing the node $i$. In such operation, one exploits the connectivity array relating the local and global index numbering for the nodes of each element. This array, in practice, defines the Boolean matrix $\left[\mathbf{B}_{i}^{H}\right]$.

In the PCG, the final solution is achieved through a sequence of matrix-vector multiplications involving the global stiffness matrix $\mathbf{K}$. One nodal component of $\mathbf{K x}$, with $\mathbf{x}$ here representing a generic vector, is given by

$$
\{\mathbf{K} \mathbf{x}\}_{i}=\sum_{H \supset i}\left[\mathbf{B}_{i}^{H}\right]\left[\mathbf{K}^{H}\right]\left\{\mathbf{x}^{H}\right\}
$$

and calculated like the residual, that is by looping over the elements. The formal analogy between (9) and (10) reflects a physical interpretation associated with the matrix-vector multiplication. Regardless of the meaning associated with $\mathbf{x}$, any operation $\mathbf{K x}$ can be interpreted as a set of forces: more precisely, the set of forces produced on the entire structure $\mathbf{K}$ by a set of fictitious displacements equal to $\mathbf{x}$. Solving the linear system (8) assumes therefore an obvious meaning: finding the deformation field $\mathbf{p}$ of the structure $\mathbf{K}$ inducing on each internal node $i$ a force $\{\mathbf{K} \mathbf{p}\}_{i}$ that offsets the action $\{\mathbf{R}\}_{i}$ produced by the imposed boundary displacement field $\mathbf{u}_{\mathbf{B}}$. This interpretation will be exploited in $\S 4$ to characterize the behaviour of the sliding boundaries.

In the context of the computational efficiency, an important issue is the choice of a good preconditioner for the conjugate gradient method, as recently confirmed in the study of Markou et al. [10]. In our work, the method was integrated within a CFD code, using the existing data structure and parallel framework OPLUS (Oxford Parallel Library for Unstructured Solvers) [18, 19]. Therefore, the parallelization of the method has been subjected to the restrictions imposed by the OPLUS library. Algorithms which are out of the scope of OPLUS are order dependent relaxation methods, such as Gauss-Seidel or LU decompositions. Because of these limitations, the Jacobi preconditioner was used throughout. 

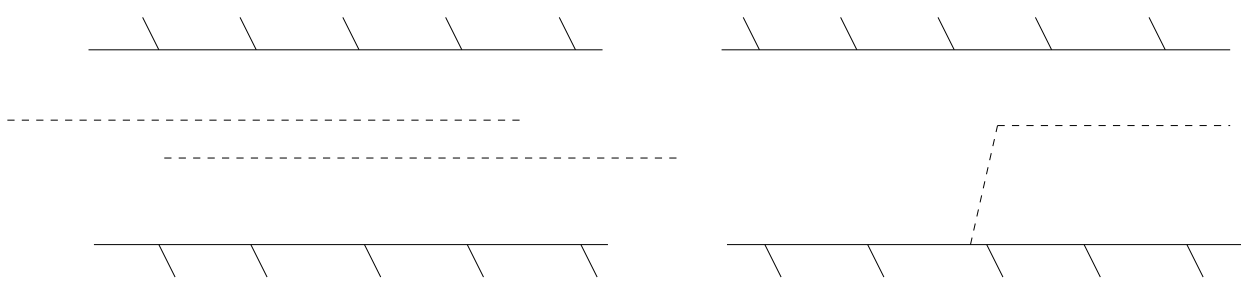

Figure 1. (a): Parallel boundaries subjected to relative motion in the boundary direction. $(b)$ : Boundary deformation forming a steep ramp.

\section{SLIDING TECHNIQUE}

In the most general case, the displacements of the moving boundaries are input parameters, and kept fixed when the new mesh is computed. However, there are some applications in which one may wish to leave more degrees of freedom to the grid. For example, a situation often encountered with artificial boundaries, such as a plane of symmetry, is when the displacements are required to be tangential to the original boundary. In this case, the identity (6) is replaced by the equation

$$
\mathbf{u}_{\mathbf{B}} \cdot \mathbf{n}=0,
$$

where $\mathbf{n}$ indicates the local normal to the boundary. Such a condition implies that the boundary nodes should not change the geometric description of the surface, but simply move remaining attached to it. A generalization of this concept can be to constrain the boundary node motion on the surface deformed by the prescribed boundary displacements $\mathbf{u}_{\mathrm{B}}$. This leads to the introduction of "sliding" boundaries, defined as those on which mesh nodes are free to move over the deflected shape.

Consider for example the situation depicted in fig. 1- $a$, with two parallel boundaries subjected to a relative movement in the boundary direction. When the relative movement becomes comparable with the distance between the boundaries, it would be convenient to cluster the nodes over one, or both, the two surfaces to maintain a limited level of skewness for the mesh elements. An other example is given in fig. 1- $b$. Here the lower boundary is folded to form a steep ramp. If the ramp develops on an axial distance comparable with the axial grid spacing, a circumstance formally described as a discontinuity of the deflection field, the moving mesh algorithm must necessarily determine a redistribution of the boundary points, so as to guarantee a proper discretization of the ramp.

The idea that we propose is to let the displacements of the boundary nodes be determined by the resolution of a modified elastic problem, in which the equilibrium is enforced in a plane tangential to the deformed surface. In general, the elastic deformation driven by the modified problem leads the points to detach from the surface. Therefore, a projection operation is needed in as subsequent stage to preserve geometric consistency. Integrated within an iterative process for geometric nonlinearity, the solution is expected to produce an adaptive distribution of the boundary nodes, more suitable to define the global deformation of the domain. 


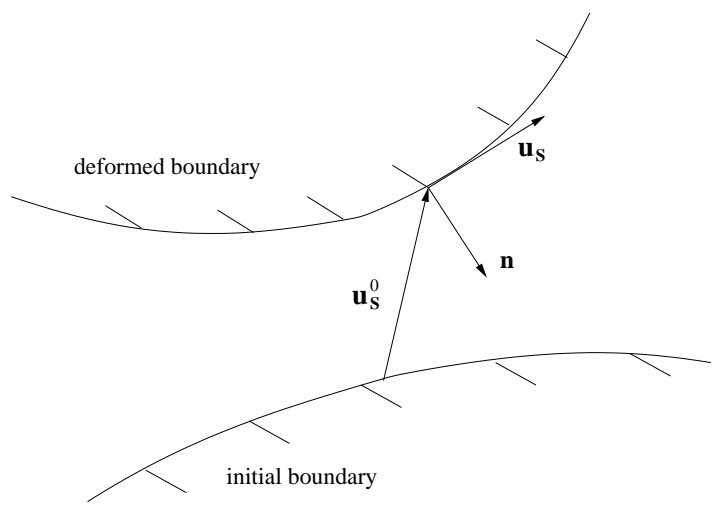

Figure 2. Displacement decomposition for a node lying on a sliding boundary.

\subsection{Formulation}

Let us consider the local equilibrium for an internal point $i$. As clarified in section $\S 3$, this corresponds to a nodal component of equation (8):

$$
\{\mathbf{K}(\mathbf{u}) \mathbf{p}\}_{i}=-\{\mathbf{R}(\mathbf{u})\}_{i} .
$$

For points on a sliding boundary, the nodal displacement is decomposed as

$$
\mathbf{u}=\mathbf{u}_{\mathbf{s}}^{0}+\mathbf{u}_{\mathbf{s}}
$$

with $\mathbf{u}_{\mathrm{s}}^{0}$ being the prescribed displacement and $\mathbf{u}_{\mathbf{s}}$ an additional degree of freedom (see fig. 2). Thus, at the $n$-th Newton-Raphson iteration one has

$$
\{\mathbf{p}\}_{i}=\mathbf{u}_{\mathbf{s}}^{(n+1)}-\mathbf{u}_{\mathbf{s}}^{(n)},
$$

with the initial value for $\mathbf{u}_{\mathbf{s}}$ chosen to be zero:

$$
\mathbf{u}_{\mathbf{s}}^{(0)}=0
$$

Now we replace the local equilibrium defined in (12) by

$$
\{\mathbf{K} \mathbf{p}\}_{i}-\left(\{\mathbf{K} \mathbf{p}\}_{i} \cdot \mathbf{n}\right) \mathbf{n}=-\mathbf{F}_{\mathbf{t}}
$$

in which $\mathbf{n}$ is the local normal to the deformed surface, and $\mathbf{F}_{\mathbf{t}}$ is defined as:

$$
\mathbf{F}_{\mathbf{t}}=\{\mathbf{R}\}_{i}-\left(\{\mathbf{R}\}_{i} \cdot \mathbf{n}\right) \mathbf{n}
$$

$\mathbf{F}_{\mathbf{t}}$ represents the tangential component of the force induced on $i$ by the imposed boundary displacement field $\mathbf{u}_{\mathrm{B}}$. The global equilibrium (8), modified for the sliding points according to (16), has not unique solution, because there exist different forces $\{\mathbf{K p}\}_{i}$ having the same tangential component equal to $-\mathbf{F}_{\mathbf{t}}$ (see fig. 3). Among the possible solutions, we select that satisfying the 


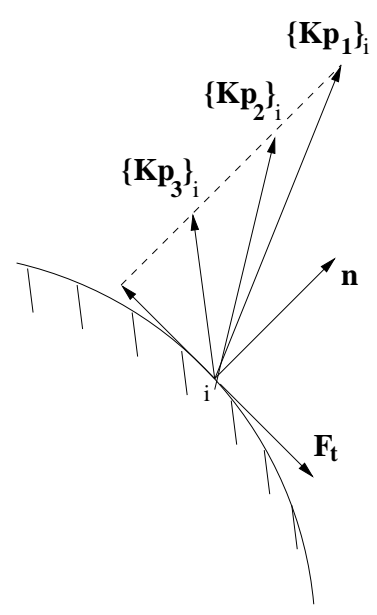

Figure 3. Non-uniqueness for the solution of the elastic problem specified by eq. (16).

kinematic condition $\mathbf{u}_{\mathbf{s}} \cdot \mathbf{n}=0$. To achieve this, the solution method described in $\S 3$ requires a little modification: in the subroutine performing the matrix-vector product, after the main loop over the elements has been performed, a further loop over the sliding points corrects the result by removing the normal component to the boundary, as indicated in (16). In the conjugate gradient method, the solution of the linear system $\mathbf{K} \mathbf{p}=-\mathbf{R}$ is searched moving along a set of conjugate directions $\mathbf{d}^{(0)}, \mathbf{d}^{(1)} \ldots \mathbf{d}^{(n-1)}$, which are sequentially determined from the residuals. In practice, one first initializes the residual and the search direction using an arbitrary value of the solution vector: $\mathbf{r}^{(0)}=\mathbf{K} \mathbf{p}^{(0)}+\mathbf{R}$, and $\mathbf{d}^{(0)}=\mathbf{r}^{(0)}$. Then, at each internal or sliding mesh node, the following steps are iterated for $k=0,1 \ldots n-1$ :

1. Update the solution

$$
\left\{\mathbf{p}^{(k+1)}\right\}_{i}=\left\{\mathbf{p}^{(k)}\right\}_{i}+\alpha^{(k+1)}\left\{\mathbf{d}^{(k)}\right\}_{i} .
$$

2. Compute the current residual

$$
\left\{\mathbf{r}^{(k+1)}\right\}_{i}=\left\{\mathbf{r}^{(k)}\right\}_{i}+\alpha^{(k+1)}\left\{\mathbf{K ~ d}^{(k)}\right\}_{i} .
$$

3. Update the search direction

$$
\left\{\mathbf{d}^{(k+1)}\right\}_{i}=\left\{\mathbf{r}^{(k+1)}\right\}_{i}+\beta^{(k+1)}\left\{\mathbf{d}^{(k)}\right\}_{i} .
$$

When the sequence above is applied to a sliding node, the initial residual and search direction are given by:

$$
\left\{\mathbf{d}^{(0)}\right\}_{i}=\left\{\mathbf{r}^{(0)}\right\}_{i}=\left\{\mathbf{K} \mathbf{p}^{(0)}\right\}_{i}+\mathbf{F}_{\mathbf{t}}
$$

Thus, since $\mathbf{F}_{\mathbf{t}}$ is parallel to the boundary, by choosing an initial value $\mathbf{p}^{(0)}=0$ one has:

$$
\left\{\mathbf{d}^{(0)}\right\}_{i} \cdot \mathbf{n}=\left\{\mathbf{r}^{(0)}\right\}_{i} \cdot \mathbf{n}=0
$$


(a)

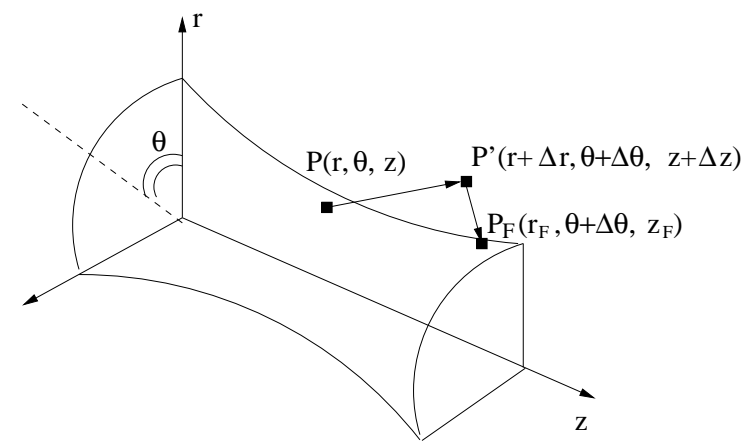

(b)

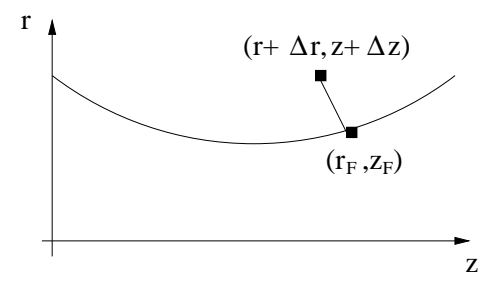

Figure 4. Correction procedure applied to sliding nodes. $(a)$ : The node slides over the axisymmetric surface. $(b)$ : The projection on a B-Spline curve is executed in the meridional plane containing $\mathbf{P}$ '.

Now, if the matrix-vector product is corrected by removal of the normal component to the boundary, the above normality conditions must necessarily be preserved, because from the steps 2 and 3 of the algorithm, one readily gets:

$$
\begin{aligned}
& \left\{\mathbf{r}^{(k+1)}\right\}_{i} \cdot \mathbf{n}=\left\{\mathbf{r}^{(k)}\right\}_{i} \cdot \mathbf{n}, \\
& \left\{\mathbf{d}^{(k+1)}\right\}_{i} \cdot \mathbf{n}=\left\{\mathbf{r}^{(k+1)}\right\}_{i} \cdot \mathbf{n}+\beta^{(k+1)}\left\{\mathbf{d}^{(k)}\right\}_{i} \cdot \mathbf{n} .
\end{aligned}
$$

Hence, provided that the initial value for $\mathbf{p}$ is chosen to be zero, the final solution resulting from the projected conjugate gradient algorithm not only satisfies the dynamic condition (16), but also guarantees that $\{\mathbf{p}\}_{i} \cdot \mathbf{n}=0$, and therefore, considering (14) and (15), the kinematic condition $\mathbf{u}_{\mathbf{s}} \cdot \mathbf{n}=0$.

We wish to point out that the ability to enforce a condition of hybrid type represents the main asset of the method. On the one hand, the dynamic condition serves to transfer the deformation of the internal nodes to the sliding boundary points, which are expected to move accordingly. On the other hand, the kinematic constraint acts in the opposite direction, converting the prescribed boundary deformation into a traction applied to the internal nodes.

\subsection{Shape reconstruction}

The projection operation is not a trivial task because it requires the reconstruction of the deformed shape, and the boundary deflection field is available in unstructured format. Inspired by the work of Samareh \& Bathia [24], we have used a CAD-based approach. The CAD model corresponding to the initial, undeformed geometry is exported by the CAD system as a set of B-Splines (or NURBS) surfaces. This is a capability that most, if not all, CAD systems provide. B-Splines are parametric surfaces of the form $\mathbf{S}=\mathbf{S}(t, s)$. Each source point $\hat{\mathbf{x}}$ from which the deflection needs to be transfered (generally from a FEM code for structural analysis), is projected onto the B-Spline surface of the undeformed geometry. In the B-Spline terminology, this latter operation is equivalent to finding the two parametric coordinates $(\hat{t}, \hat{s})$ such that $\hat{\mathbf{x}}=\mathbf{S}(\hat{t}, \hat{s})$. At this point, a B-Spline of the deformed boundary is constructed by global interpolation of the deflection data, performed in the parametric space $(t, s)$. 


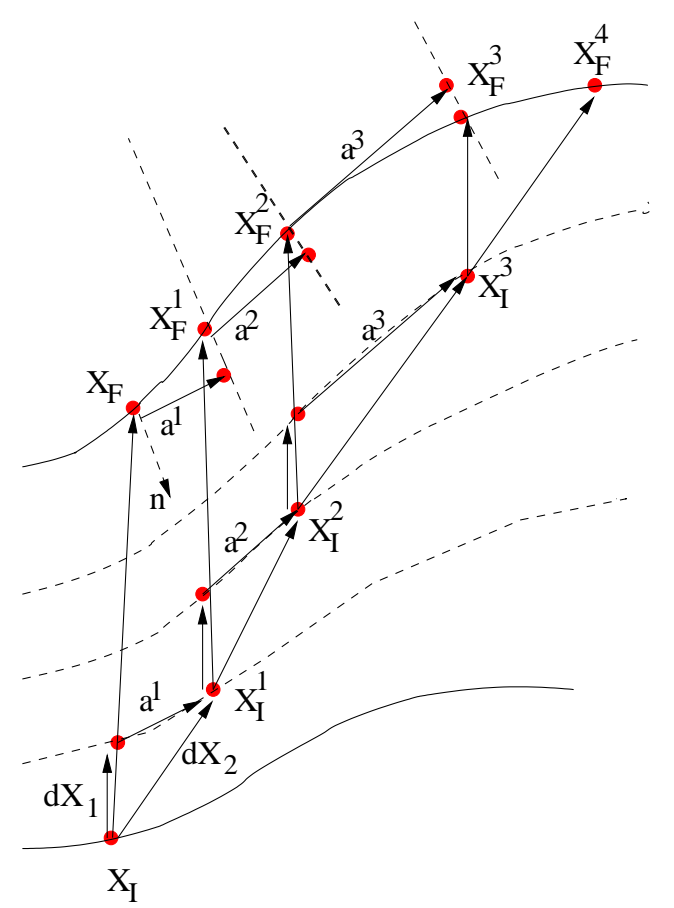

Figure 5. Schematic evolution of a point lying on a sliding boundary, subjected to a deformation divided in 4 ranges.

In our application the task has been facilitated by the fact that, although the mesh to update is three-dimensional, the deforming surfaces represent an axisymmetric geometry, and are subject to axisymmetric deflections. As better clarified in $\S 6$, this results from the use of a methodology coupling an axisymmetric FEM model to a three-dimensional CFD model. Therefore, the surface that we need to reconstruct is generated by rotating a curve $\mathbf{c}$ around an axis, for an angle corresponding to the rotational periodicity of the CFD model. In a cylindrical frame of reference aligned with the axis of rotation (see fig. 4-a), a sliding node $\mathbf{P}(r, \theta, z)$ moves initially to $\mathbf{P}^{\prime}(r+\Delta r, \theta+\Delta \theta, z+\Delta z)$ due to the sliding mechanism. To project $\mathbf{P}^{\prime}$ on the surface, we perform 2D operations in the meridional plane containing $\mathbf{P}^{\prime}$. In such a plane (fig. 4-b), the point having coordinates $(r+\Delta r, z+\Delta z)$ is projected on the curve $\mathbf{c}$ to give $\left(r_{F}, z_{F}\right)$. The position of $\mathbf{P}$ is finally set equal to $\mathbf{P}_{\mathbf{F}}\left(r_{F}, \theta+\Delta \theta, z_{F}\right)$.

We now address the question of how to get an analytical expression for the curve $\mathbf{c}$ in order to carry out the projection in the meridional plane. The intersection of a CAD surface with a meridional plane is a B-Spline curve of the form

$$
\mathbf{r}(t)=\sum_{i=0}^{n} N_{i}^{p}(t) \mathbf{P}_{i} \quad 0 \leq t \leq 1,
$$

where $\mathbf{P}_{i}$ are the control points, and $N_{i}^{p}(t)$ are the $p$-th degree B-Spline basis functions. Thus, once the initial CAD model has been completed, one has available a B-Spline description $\mathbf{r}(t)$ of the curve generating, through an appropriate rotation, the surface of the initial geometry.

In the axisymmetric FEM model, the boundary nodes $\mathbf{Q}_{1}, \mathbf{Q}_{2} \ldots \mathbf{Q}_{k}$ with the computed deflections $\delta_{1}, \delta_{2} \ldots \delta_{k}$, correspond to points lying on the curve $\mathbf{r}(t)$. Therefore, the set of nodes can be reordered 


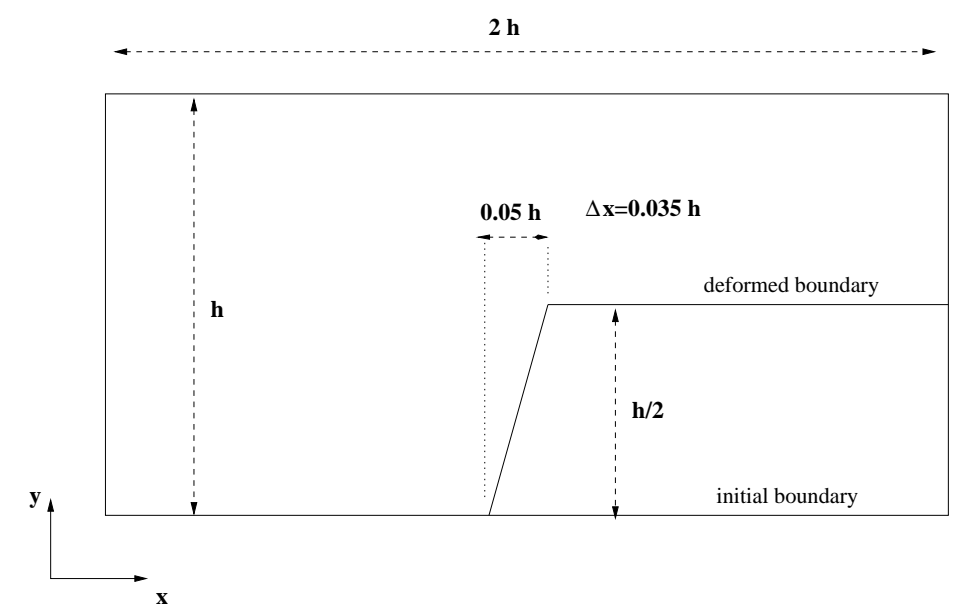

Figure 6. Definition of the $2 \mathrm{D}$ test case. In a box $h \times 2 h$ the lower boundary is folded to create a ramp developing on an axial distance comparable with the axial grid spacing $\Delta x$ of the initial mesh.

according to the value of the parameter $t$, retrieved from the projection of each point $\mathbf{Q}_{j}$ on $\mathbf{r}(t)$. The ordering of data recovers the point to point connectivity of the curve designed in the CAD system, thereby converting into a structured format the unstructured set of data. After that, a BSpline interpolant for the deflected curve $\mathbf{c}(t)$ is obtained by setting up the linear system

$$
\mathbf{Q}_{j}+\delta_{j}=\sum_{i=1}^{k} N_{i}^{q}\left(t_{i}\right) \mathbf{T}_{i} \quad j=1 \ldots k
$$

in which the unknowns $\mathbf{T}_{i}$ are the control points of the $q$-th degree B-Spline describing the deflected curve c. The basis functions $N_{i}^{q}$ have compact support, and so the ordering of data based on the value of $t$ ensures that the system (21) is narrow banded, and hence quickly invertible by LU decomposition. The choice of the degree $q$ should be consistent with the discretization order employed in the FEM code. The curve $\mathbf{c}(t)$ is of class $C^{(q-1)}$, therefore, assuming $q=1$ one recovers a linear behaviour for the element deformation in the FEM model.

The above set of operations is carried out only once, by each individual processor, immediately after reading the deflection data. Then, while the new mesh is being computed, the projection is executed at the end of every Newton-Raphson iteration and involves all the sliding points pertaining to each partition. The problem of projecting a point to the curve $\mathbf{c}(t)$ is solved using Newton iterations, searching the value of the parameter $t$ that minimizes the distance between the point and the curve [25]. These can be efficiently executed using the De Boor algorithm [25], which allows a quick evaluation of $\mathbf{c}(t)$ and its derivatives, for a given value of $t$.

\subsection{Re-start procedure}

This section focuses on technical aspects concerning the applicability of the method to a deformation progressively changing during a coupled analysis. A prerequisite for an efficient use of the scheme, in fact, is the ability to update the mesh with respect to the partial deflections prescribed in input. To achieve this, some care has to be taken if the sliding technique is employed. 
(a)

(b)
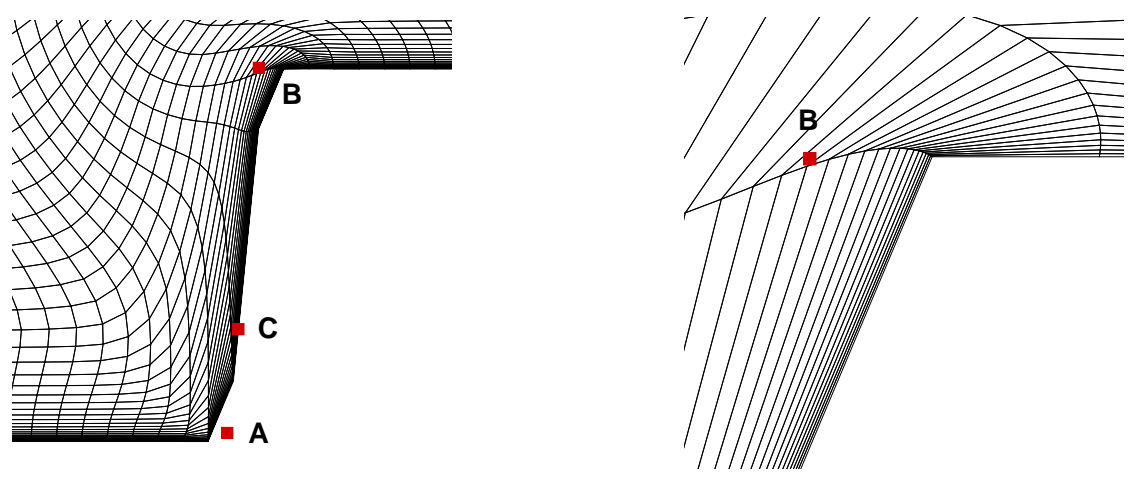

(c)

(d)
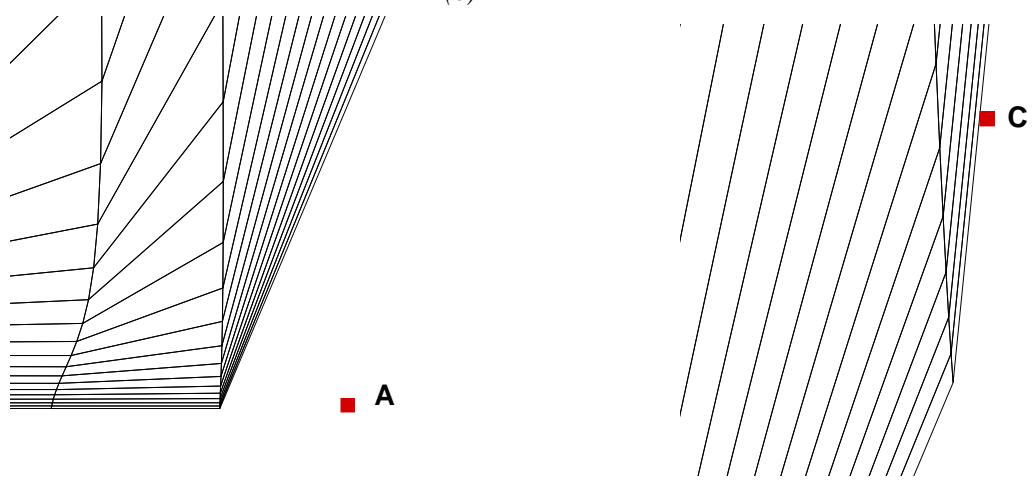

Figure 7. 2D test case. Mesh computed without the sliding technique. (a): Global view of the ramp. (b): Close-up view at the upper corner B. (c): Close-up view of the lower corner A. (d): Close-up view at the point C, located in the ramp.

Figure 5 shows the evolution of a point lying on a sliding boundary subjected to a displacement divided into 4 ranges. Dotted lines represent the geometry corresponding to each sub-deflection. For the time being, let these be artificial increments deliberately introduced to facilitate the convergence of the algorithm. The case with the displacements deriving from a transient deformation of the solid part is a straightforward extension of the considerations developed here.

The point $\mathbf{x}_{\mathbf{I}}$ is assigned a vector displacement $\mathbf{x}_{\mathbf{F}}-\mathbf{x}_{\mathbf{I}}$. Starting from the first mesh increment, the algorithm accommodates the initial sub-deflection $\mathbf{d x}_{1}$, moving the point along the partial geometry. This operation provides a modified value $\mathbf{d x}_{2}$ for the sub-deflection. The difference between the two vectors $\mathbf{a}_{\mathbf{1}}=\mathbf{d x}_{\mathbf{2}}-\mathbf{d x}_{\mathbf{1}}$, is the slide vector produced by the algorithm on the partial geometry. Before resuming the computation on the next increment, the slide vector $\mathbf{a}_{1}$ is added to $\mathrm{x}_{\mathbf{F}}$ and the result projected on the boundary corresponding to the complete deformation, to give $\mathrm{x}_{\mathrm{F}}^{1}$. At the successive stage, the scheme is let to run on a displacement given by:

$$
\delta=\frac{1}{3}\left[\mathbf{x}_{\mathbf{F}}^{1}-\left(\mathbf{x}_{\mathbf{I}}+\mathbf{d x}_{\mathbf{2}}\right)\right] .
$$


(a)

(b)
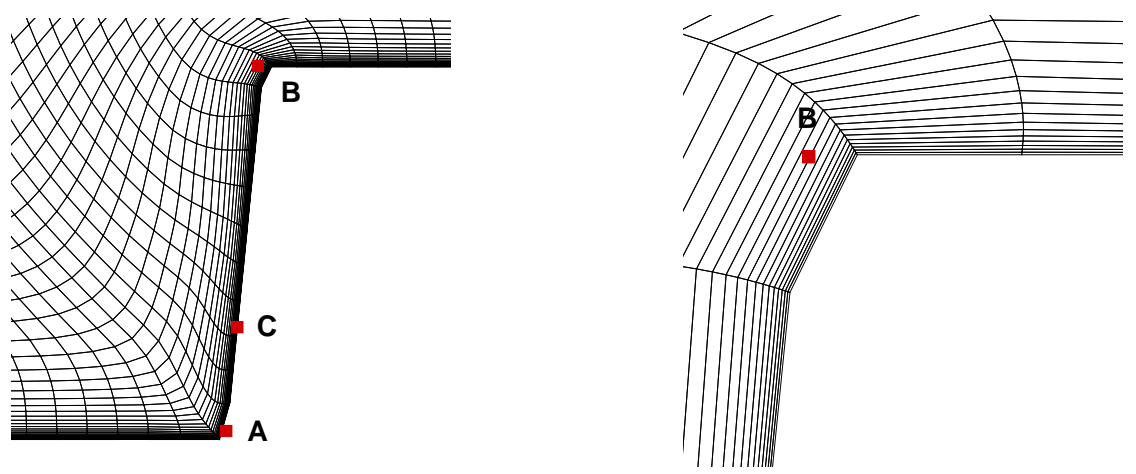

(c)

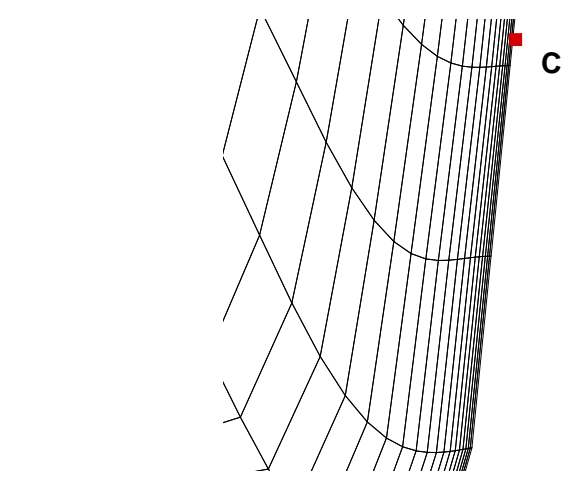

(d)

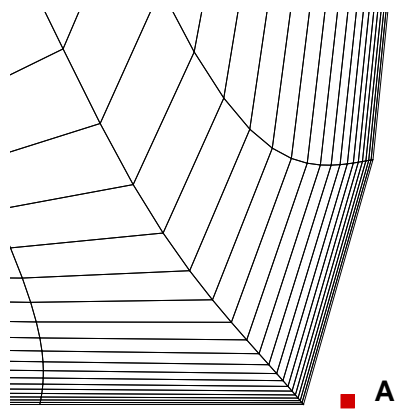

Figure 8. 2D test case. Mesh computed with the sliding technique. (a): Global view of the ramp. (b): Close-up view at the upper corner B. (c): Close-up view of the lower corner A. $(d)$ : Close-up view at the point C, located in the ramp.

Thus, as the point $\mathbf{x}_{\mathbf{I}}$ gradually reaches its final destination through the sequence $\mathrm{x}_{\mathbf{I}}^{1}, \mathbf{x}_{\mathbf{I}}^{2}, \mathbf{x}_{\mathbf{I}}^{3}$, the displacements driving the elastic response are sequentially adjusted to $\left(\mathbf{x}_{\mathbf{F}}^{1}-\mathbf{x}_{\mathbf{I}}^{1}\right) / 3,\left(\mathbf{x}_{\mathbf{F}}^{2}-\mathbf{x}_{\mathbf{I}}^{2}\right) / 2$ and $\left(\mathrm{x}_{\mathbf{F}}^{3}-\mathrm{x}_{\mathbf{I}}^{3}\right) / 1$. This procedure guarantees an appropriate nodal displacement as input to the mesh moving algorithm. Conversely, if the partial deflections are permanently computed with respect to the assigned value $\mathbf{x}_{\mathbf{F}}$, the method could struggle to convergence at the latest steps, because the final sub-deflections can become too severe. This, in practice, would cancel the benefit that one wishes to gain by splitting the displacements in several increments. Similar considerations can be carried out in the case of re-start from a previous analysis, the only difference being that, now, the adjustment of the forcing displacement has to be done once, beforehand, and exploiting the slide vector $\mathbf{a}$ associated with the previous analysis.

\section{TWO-DIMENSIONAL TEST CASE}

The test case discussed in this section reproduces the example illustrated in fig. 1- $b$. In a rectangular domain with height $h$ (see fig. 6), the lower boundary is folded to form a steep ramp in the centre. 


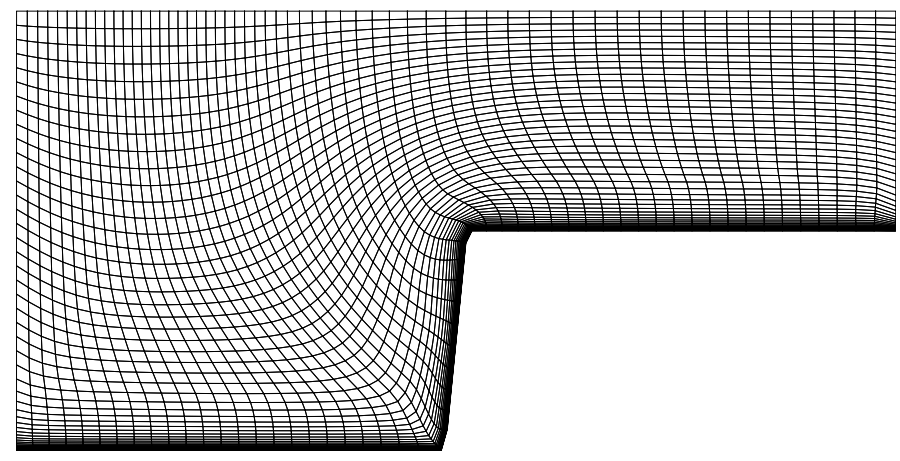

Figure 9. 2D test case. Global view of the mesh obtained applying the sliding technique for the lower and upper boundary.

The ramp has height $h / 2$, and develops linearly on an axial distance of $0.05 h$. The rectangular mesh has a uniform axial grid spacing of $\Delta x=0.035 h$, and is strongly stretched in the y-direction to represent a more challenging boundary layer mesh. This is an example of discontinuity in the deflection field, in the sense that there exist two contiguous boundary nodes, at a distance $\Delta x$, undergoing a displacement $\mathbf{u}$ such that $\Delta \mathbf{u}>>\Delta x$. The final configuration is achieved using 16 increments. For each of them, the initial residual of the Newton-Raphson cycle is reduced by a factor of $10^{-4}$ by successive calls to the linear solver.

Figure 7 shows the mesh obtained using the standard structural analogy, with the sliding option turned off. The method is not unsuccessful and generates the mesh one would theoretically expect: in fact, the inter-penetration of neighboring cells is avoided. Note that the distribution of the boundary nodes does not preserve the corners of the ramps (denoted in the figure by the points $\mathbf{A}$ and $\mathbf{B}$ ). Clearly, this is because the ends of the ramp have been located arbitrarily, and in the initial mesh there are no boundary nodes placed on the two folding points. This reflects a situation in which one doesn't know, a priori, the location and the size of the developing discontinuity, and therefore is unable to discretize the region adequately. In the zone between the points $\mathbf{A}$ and $\mathbf{B}$ the boundary remains discretized by three cells only, and abnormal deformation of the elements must necessarily occur.

As expected, the problem is solved allowing the boundary nodes to re-distribute themselves. Figures 8 and 9 show the mesh computed applying the sliding technique to both lower and upper boundary. Boundary nodes cluster over the ramp, resulting in a drastic improvement of the mesh quality. Even in this case, the method is unable to preserve the corners. As before, this is just because the discrete description of the boundary (based on the new nodal distribution), does not have corners.

The sliding option reduces the computational efficiency of the method. In this example, the CPU time required was 2.5 times bigger, but our experience is that this factor is largely dependent on the specific case under investigation. The increase is mainly $(\sim 75 \%)$ due to a degraded convergence rate for the Newton-Raphson cycle, which, in practice, requires more iterations to find 


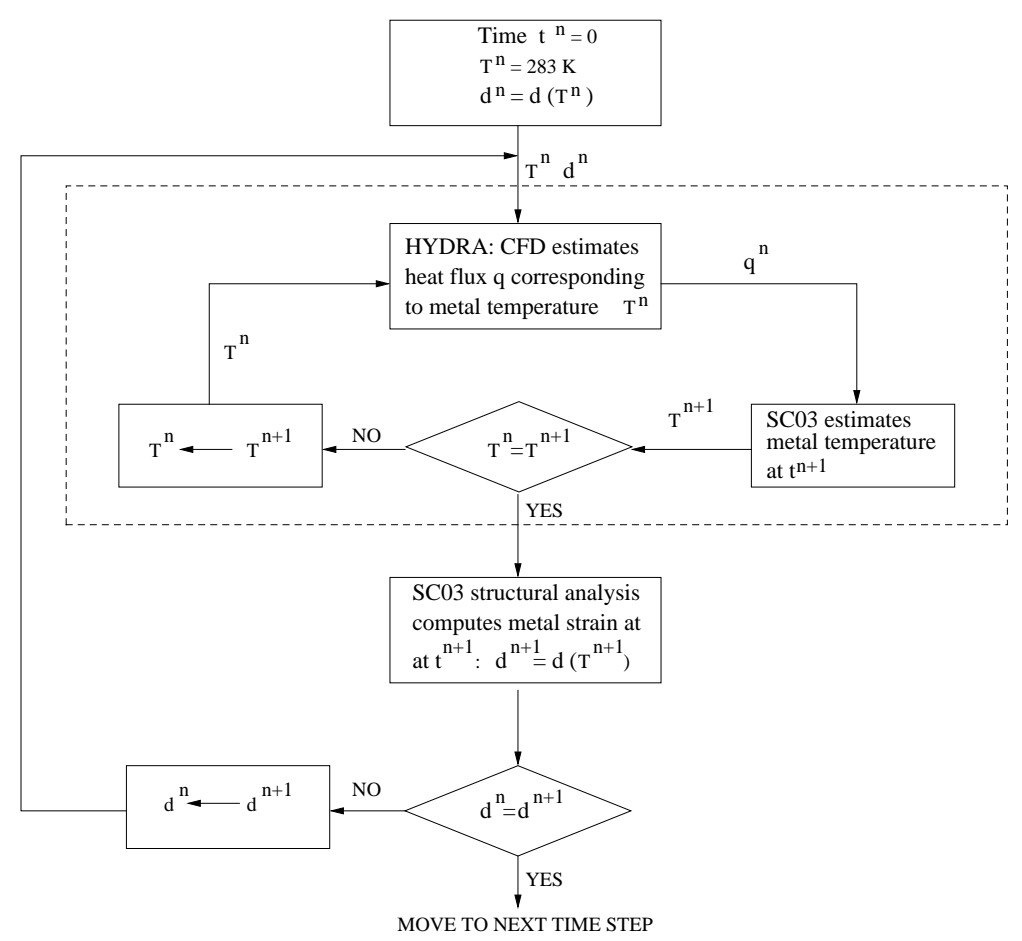

Figure 10. Schematic representation of the coupling process.

the equilibrium configuration; about $15 \%$ is taken by the PCG solver, since the system to invert has additional degrees of freedom, while the remaining $10 \%$ corresponds to the extra work required for the projections. These considerations suggest limiting the use of the technique to the boundaries of the most critical regions, whenever necessary. In realistic applications, these may cover a limited portion of the entire domain, so that the loss of efficiency is expected to be tolerable.

\section{CAVITY INTERSTAGE OF A TURBINE}

The application described in this section refers to the aero-thermo-mechanical analysis of an existing engine component. In order to clarify the use made of the dynamic mesh, it is convenient, before describing results, to give an overview of the coupling methodology, with a description of the model set-up.

\subsection{Model set-up}

The coupled analysis has been conducted using two distinct Rolls-Royce proprietary codes, SC03 and HYDRA. SC03 is a finite element code performing transient thermal and mechanical analysis [26]. HYDRA [27] is an unstructured finite volume solver of the compressible Reynolds Averaged Navier Stokes equations.

A schematic description of the coupling process is given in fig. 10. Within $\mathrm{SC} 03$, the user defines a transient analysis by specifying a "flight cycle", i.e. the evolution of a set of parameters through 


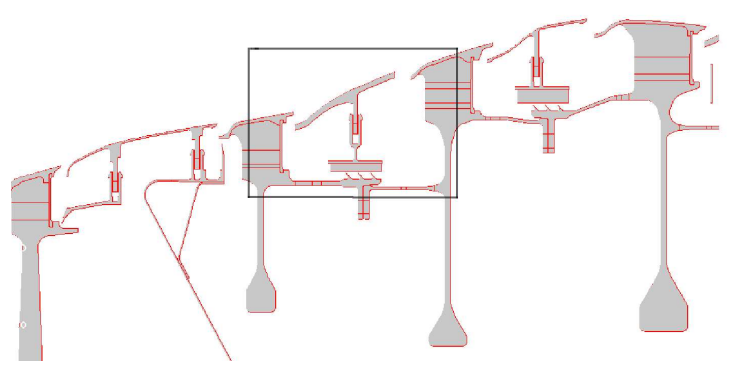

Figure 11. Geometry of the turbine. Enclosed in the square is the stator well where the coupling takes place.
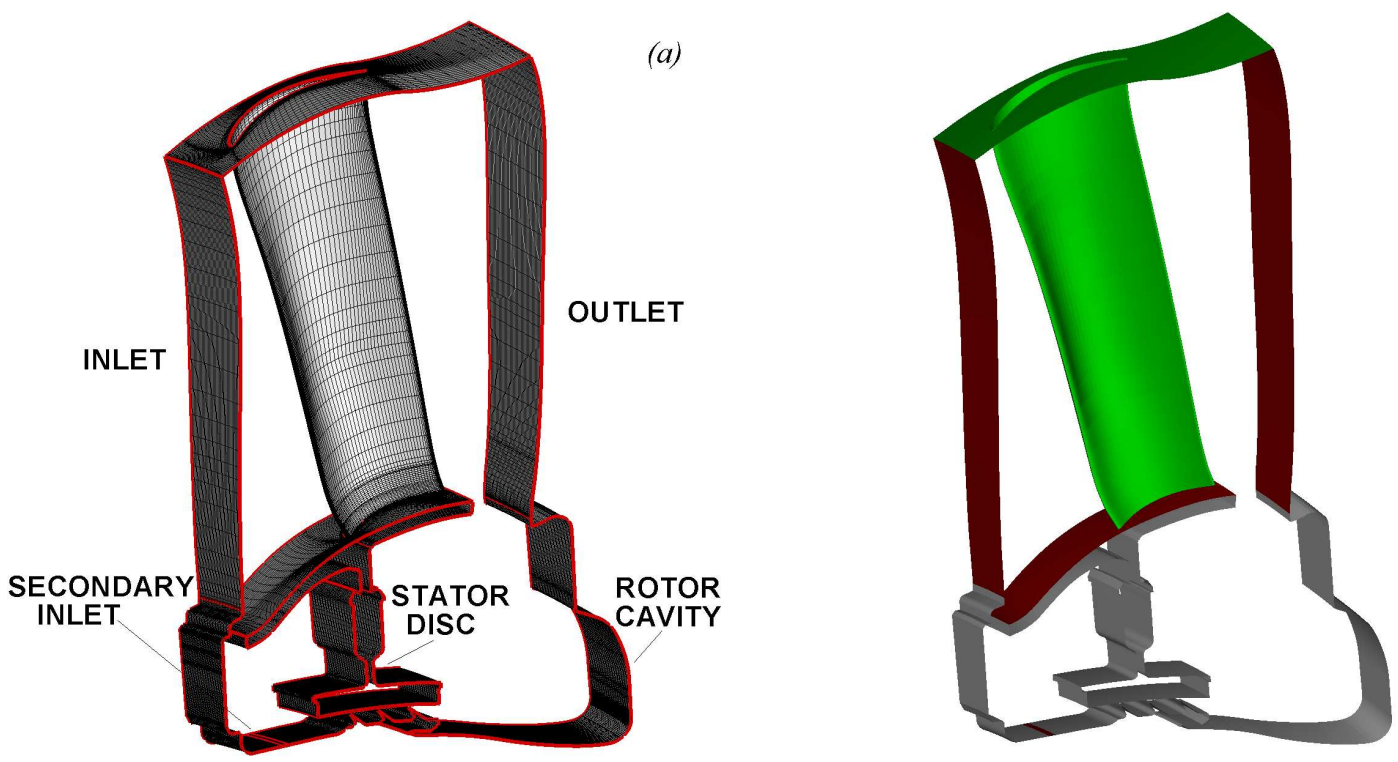

Figure 12. CFD model. (a): geometry and initial mesh. $(b)$ : surface definition in the mesh deformation algorithm. Green: fixed boundaries. Red: passive boundaries. Grey: boundaries with prescribed displacements.

the time span to simulate. These include rotational speed, mass flow rates, operating temperatures and pressures. At each time point during the cycle, the coupling methodology employs two nested iterative loops. The external one couples the thermal and structural analysis; these are performed in sequence by SC03 until both geometry and temperature are stabilised. The internal loop is called during the thermal analysis: the system invokes the CFD code HYDRA, passing to it the current estimate of boundary temperatures and boundary deflections.

Since the time response of the flow is negligible on the time scale relative to the metal heat conduction, HYDRA adapts an initial mesh to conform to the boundary deflections, and runs a steady state case with the prescribed wall temperatures. The resulting heat fluxes are returned to SC03, which in turns repeats the thermal analysis on the same geometry but using the CFD-based values of heat flux. When the difference in metal temperature between two consecutive thermal iterations is below a specified tolerance, the external loop resumes. Typically two or three external iterations are required for convergence at each time step, after which the analysis moves to the next time step in the cycle, corresponding to the next level in the temporal discretization of the 


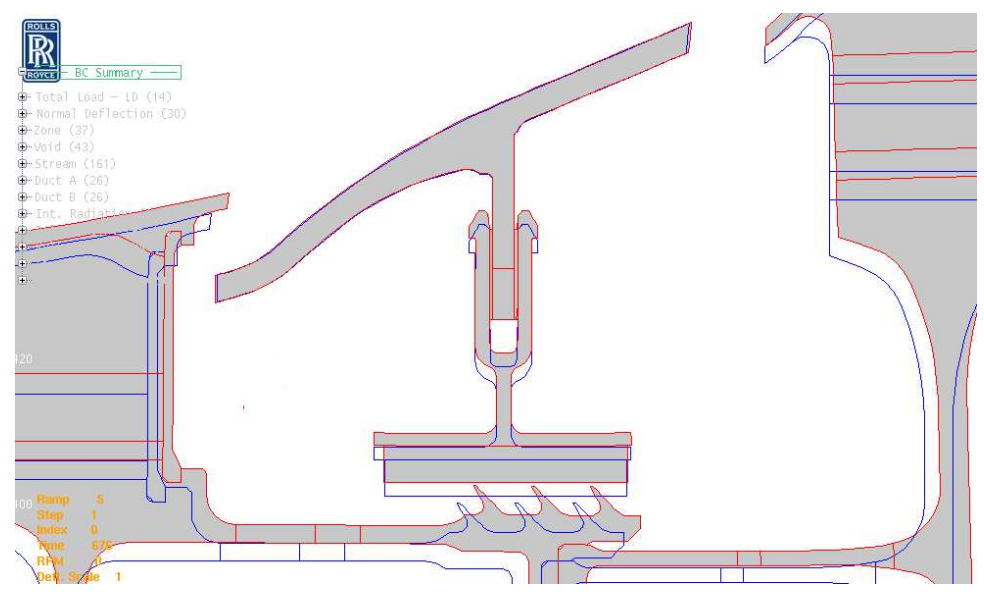

Figure 13. Metal deformation predicted by SC03. Blue lines identify the undeflected shape.

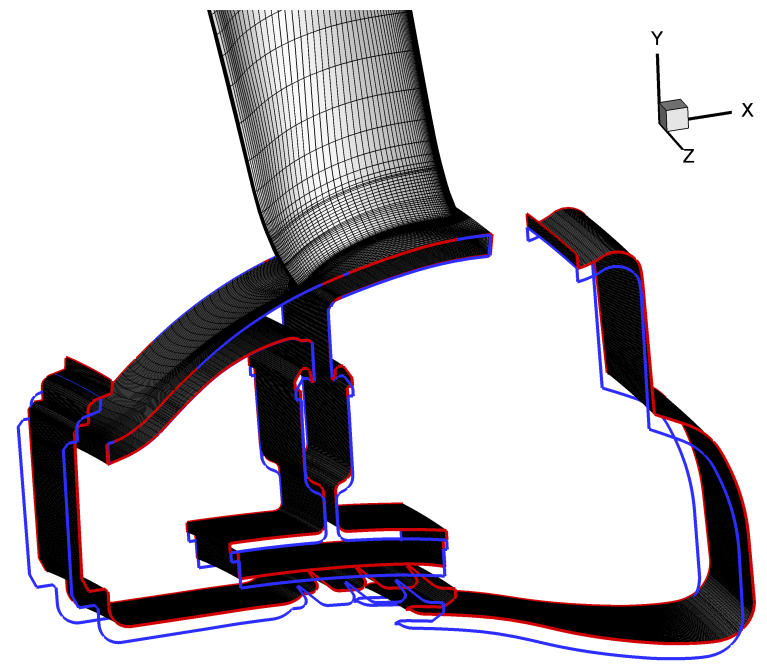

Figure 14. Fluid domain deformation corresponding to the displacement field of fig. 13. Blue lines identify the undeflected shape.

heat conduction equation. Thus, in the process described, the re-meshing frequency depends on the integration time step of the metal heat conduction.

Figure 11 shows the SC03 axisymmetric model of the turbine under investigation. Enclosed in the square is the stator well coupled with the CFD. To render the analysis affordable, the coupling is restricted to the most critical parts, whereas for other components semi-empirical heat transfer correlations are used. These correspond to the standard boundary conditions traditionally used in industry for the thermal analysis.

The fluid domain is given in figure 12- $a$. It is a three-dimensional model with rotational periodicity of $3.6^{\circ}$, and the mesh consists of 2.7 million hexahedral cells. Note that the blade over the stator disc is not included in the axisymmetric SC03 model. As a consequence, the blade in the CFD model has to be maintained fixed during the calculation. Similarly, the inlet/outlet 

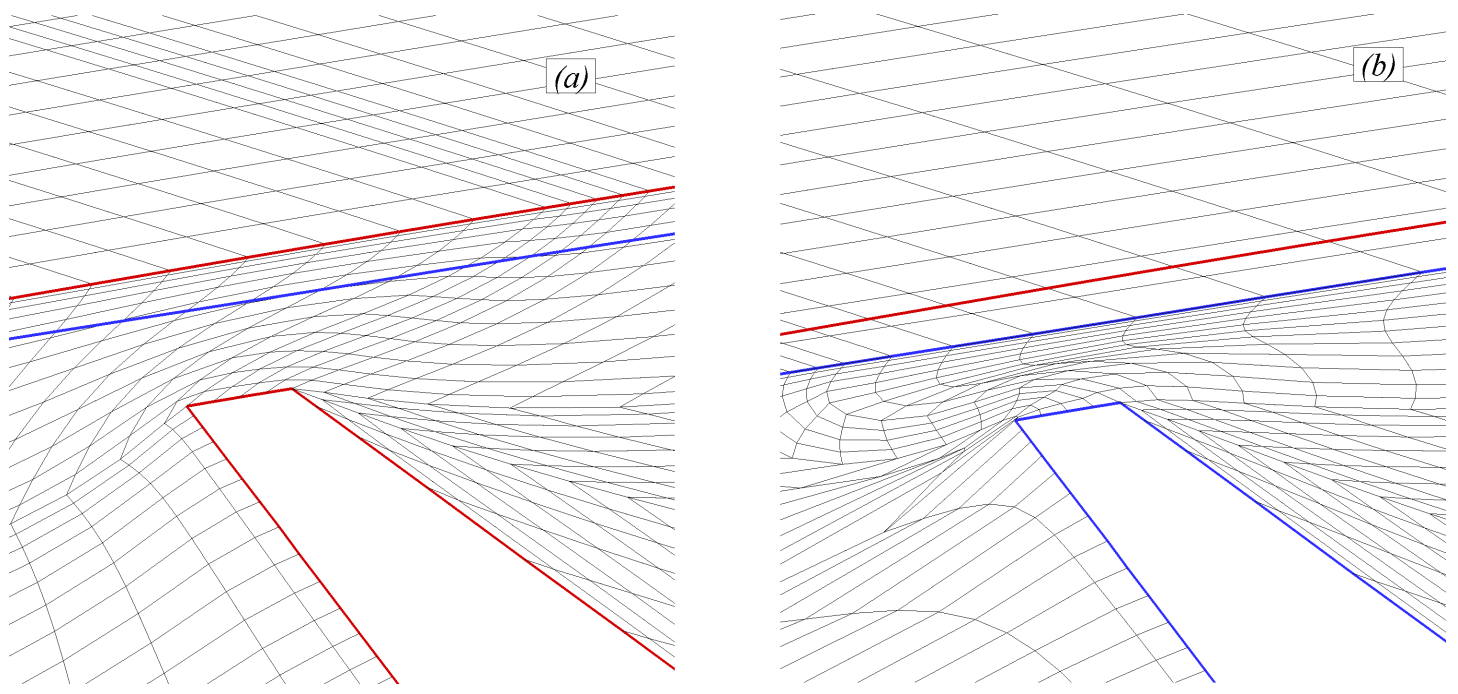

Figure 15. Close-up view of the mesh in the labyrinth seal region. $(a)$ : Sliding option on. $(b)$ : Sliding option off. Blue lines identify the outline of the surface as prescribed in input. Red lines identify the new periodic edge created by the sliding technique.

boundaries correspond to artificial surfaces for which nodal displacements are not prescribed. In the moving mesh algorithm, we define as "passive" those surfaces whose mesh nodes are not subject to a displacement from input. Figure 12- $b$ clarifies this specification: the "fixed" surfaces are the blade and the external casing. The passive surfaces include the inlet/outlet boundaries and the external surface of the stator disc. For this latter surface, although SC03 provides displacements on the interior points, the specification as a passive boundary preserves the consistency with the attached blade. The passive surfaces are treated before the volume computation, solving separate elastic problems involving only the points lying on the surface. We use fast linear spring analogy algorithms, determining the position of the nodes from the displacements imposed on the boundary of the surface. The solution of these preliminary computations provide the prescribed boundary displacements for the 3D structural analogy.

Finally, we observe that in the CFD mesh the periodic boundaries are not planar. Therefore, any 2D displacement field passed by SC03 will have one component on the periodic surface, and one component normal to it. The entire coupled analysis lasted 4 weeks running on 80 CPUs of a PC cluster. The mesh adaptation took approximately $25 \%$ of the whole computational time.

\subsection{Results}

The performance of the method is assessed by considering the effect of the most severe distortion computed during the flight cycle. A snapshot of the metal deformation, as predicted by $\mathrm{SC03}$, is given in fig. 13, while fig. 14 shows the corresponding deformation for the fluid domain. Under the effect of the mechanical loads transmitted through the shaft, the rotor cavity is subjected to both axial and radial displacements. It should be noted that lower and upper disc components are jointed together through a mechanism allowing their relative movement in the radial direction, to preserve seal clearance. 

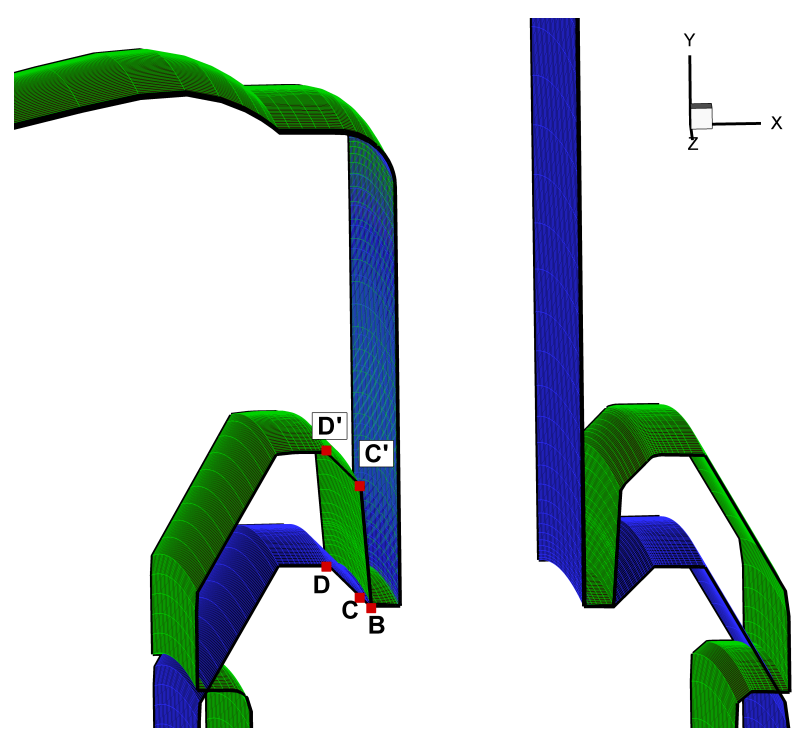

Figure 16. Close-up view of the joint region. Green surfaces identify the deformed boundaries.

The dynamic re-meshing procedure encounters its major difficulties in regions with small gaps. In the labyrinth seal, for example, the distance from stator to rotor is reduced by approximately $60 \%$. Elastic or spring analogy methods are generally adequate to handle mesh constrictions. The narrowing, in fact, does not involve angular variations of the elements. In the case discussed here, however, the problem arises because the constriction is combined with an axial translation of the cavity. It turns out that if the boundary nodes are maintained at prescribed positions, element distortions are unavoidable and may reach unacceptable levels. This is the scenario in which the sliding technique can offer a drastic improvement, as confirmed by the figure 15- $a$. The disc base is modelled as a sliding boundary, so that mesh nodes lying on it can move forward driven by the cavity movement. The final mesh is not only valid, but is also of relatively good quality. Figure 15 visualises the mesh on a periodic surface for the two cases (sliding option on/off). For the periodic nodes located on the edge of the disc base, the kinematic constraint $\mathbf{u}_{\mathbf{s}} \cdot \mathbf{n}=0$ does not prevent the points from shifting in the azimuthal direction. Consequently, the algorithm redesigns the periodic edges of the disc base. In the figure, blue lines represent the outline of the surface as prescribed in input, whereas red lines identify the new periodic edges resulting from the azimuthal shift.

Proceeding in the analysis of the deformation, we now focus on the region where lower and upper disc components are connected. Figure 16 provides a close-up perspective of the radial relative movement. The two points $\mathbf{B}$ and $\mathbf{C}$ of the undeformed geometry are initially positioned close to each other. The deformation leaves the point $\mathbf{B}$ fixed, because in the SC03 model this point is treated as belonging to the upper part of the disc, whereas the point $\mathbf{C}$ moves up to $\mathbf{C}^{\prime}$, driven by the radial excursion of the lower disc component. Consequently, the prescribed displacement field has a strong gradient between the points $\mathbf{B}$ and $\mathbf{C}$. The situation is similar to the steep ramp analysed for the $2 \mathrm{D}$ test case (cfr. $\S 5$ ), but the difficulty here is augmented by the fact that the mesh is confined to a very narrow gap.

The mesh computed is shown in fig. 17. With the sliding option turned off, the elastic analogy fails (fig. 17-b), because the edge confined between the points $\mathbf{B}$ and $\mathbf{C}^{\prime}$ is left under-resolved, 


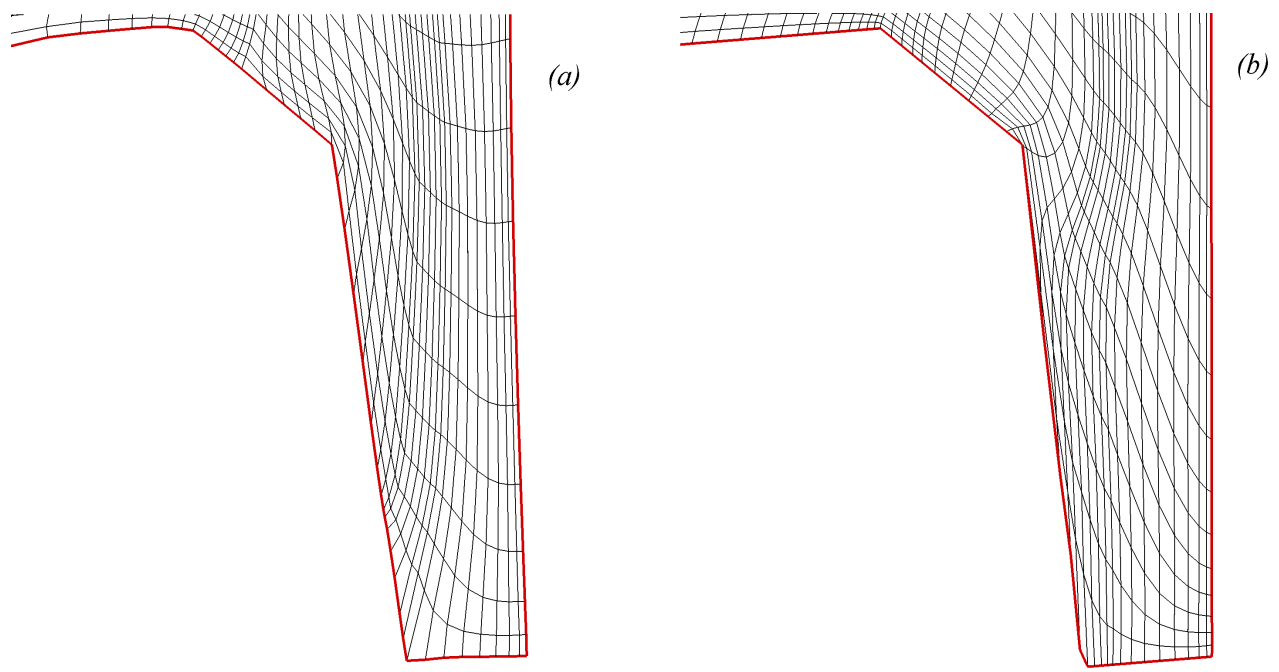

Figure 17. Close-up view of the mesh in the joint region. (a): Sliding option on. (b): Sliding option off.

and flat elements occur. Note also the interpenetration occurring at the cell near the upper corner, corresponding to the point $\mathbf{C}$ ' of fig. 16. Conversely, the sliding technique improves the distribution of boundary points, preventing the failure. The quality of the final mesh is certainly not perfect, but the result is deemed satisfactory considering the extreme difficulty of the case.

\section{CONCLUSIONS}

A modified version of the elastic medium analogy for dynamic meshes has been presented. The method relies on the inclusion of the boundary points within the elastic problem. In regions where two distinct bodies undergo strong relative motion, boundary nodes are constrained to satisfy the elastic equilibrium in the direction tangential to the surface. This leads the boundary nodes to slide over the deflected shape, i.e. the surface defined by the prescribed boundary displacements. Integrated into an iterative Newton-Raphson cycle, the method adjusts the boundary node distribution to make it more suitable to the global geometric configuration defined by the deformation.

As a test case, a three-dimensional model for a turbine stator well has been used, subject to deformations computed by a coupled 2D to 3D aero-thermo-mechanical analysis. Studies recently conducted by the authors [17] have shown the importance of a correct prediction of the running clearances for this type of application.

The sliding technique introduced is simple and completely general in the context of structural analogy methods (spring or elastic medium analogies, structured or unstructured meshes), but it needs to be combined with an appropriate methodology for the reconstruction of the moving boundaries. A variety of methods can be used, depending on the specific application and on the general structure of the codes adopted. In this paper, a CAD-based approach has been discussed: the CAD description of the original geometry is used to determine a B-Spline interpolant for the boundary deflection. The methodology is efficient, concise, and its application to the moving mesh 
algorithm presented should be extended, in the future, to cases involving deformations of blades and fans.

\section{ACKNOWLEDGEMENTS}

The present investigations were supported by the European Commission within the Framework 6 Programme, Research Project Main Annulus Gas Path Interaction (MAGPI). AST5-CT-2006-30874. This financial support is gratefully acknowledged.

\section{REFERENCES}

1. Heselhous A., Vogel D.T. and Krain H., Coupling of 3D Navier-Stokes external flow calculations and internal 3D heat conduction calculation for cooled turbine blades. AGARD 40, 1992.

2. Chew J.W., Taylor I.J and Bonsell J.J., CFD developments for turbine blade heat transfer. IMECHE Conference Transactions, Vol.1, 1996.

3. He L. and Oldfield M., Unsteady conjugate heat transfer. ASME Paper 2009-GT-51151.

4. Verdicchio J.A., Chew J.W. and Hills N.J., Coupled fluid/solid heat transfer computation for turbine discs. ASME Paper 2001-GT-0123.

5. Sun Z., Chew J., Hills N.J., Volkov K. and Barnes C.J., Efficient FEA/CFD thermal coupling for enineering applications, Journal of Turbomachinery, 2009.

6. Batina J.T., Unsteady Euler airfoil solutions using unstructured dynamic meshes. AIAA J., 28(8), 1990.

7. Farhat C., Degand C., Koobus B. and Lesoinne M., Torsional springs for two-dimensional dynamic unstructured fluid meshes. Comput. Methods Appl. Mech. Engrg., 163, 1998.

8. Johnson A.A., Tezduyar T.E., Mesh update strategies in parallel finite element computations of flow problems with moving boundaries and interfaces. Comput. Methods Appl. Mech. Engrg., 119, 1994.

9. Degand C., Farhat C., A three-dimensional torsional spring analogy method for unstructured dynamic meshes. Comput. Struct., 80, 2002.

10. Markou G.A., Mouroutis Z.S., Charmpis D.C. and Papadrakakis M.. The orto-semi-torsional (OST) spring analogy method for 3D mesh moving boundary problems. Comput. Methods Appl. Mech. Engrg., 196, 2007.

11. Bottasso C.L, Detomi D. and Serra R.. The ball vertex method: a new siple analoy method for unstructured dynamic meshes. Comput. Methods Appl. Mech. Engrg., 194, 2005.

12. Zeng D. and Ethier C.R.. A semi-torsional analogy method for updating the unstructured meshes in 3D moving domains. Finite Elem. Anal. Des., 41, 2005.

13. Johnson A., Tezduyar T., Advanced mesh generation and update methods for 3D simulations. Comp. Mech., 143, 1999.

14. Stein K., Tezduyar T. and Benney R., Mesh moving techniques for fluid-structure interaction with large displacements. J. Appl. Mechanics, 70, 2003.

15. Hsu S.Y. and Chang C.L., Mesh deformation based on fully stressed design: the method and two dimensional examples. Int. J. Numer. Meth. Eng., 72, 2007.

16. Kholodar D.B., Morton S.A., Cummings R.M., Deformation of unstructured viscous grids. AIAA J., 926, 2005.

17. Amirante D., Hills N.J. and Barnes C.J., Thermo-mechanical FEA/CFD coupling of an interstage seal cavity using torsional spring analogy. ASME Turbo Expo, GT2010-22684, 2010.

18. Crumpton P.I. and Giles M.B., Oplus Fortran 77 library, http://www.comlab.ox.ac.uk.

19. Hills N.J., Ahieving high parallel performance for unsteady turbomahinery code, The Aeronautical Journal, UK Applied Aerodynamics Consortium Special Edition, 111, 2007.

20. Smith I.M and Griffith D.V., Programming the finite element method, John Wiley and Sons.

21. Kiusalaas J., Numerical methods in engineering with Matlab, Oxford University Press, 2005.

22. Yang Z. and Mavriplis D.J., Unstructured dynamic meshes with higher-order time integration schemes for the unsteady Navier-Stokes equations, AIAA Paper 2005-1222, 2005. 
23. Biedron R.T. and Thomas J.L., Recent enhancements to the FUN3D flow solver for moving mesh applications, AIAA Paper 2009-1360, 2009.

24. Samareh J.A. and Bathia K.G., A unified approach to modeling multidisciplinary interactions, AIAA Paper 20004704, 2000

25. Piegl L. and Tiller W., The NURBS book, Springer, 1996.

26. Armstrong I. and Edmunds T.M., Fully authomatic analysis in the industrial environment, Proceedings of $2^{\text {nd }}$ international conference on quality assurance and standards, NAFEMS, 1989.

27. Monier P., Algorithm Developments for an unstructured viscous flow solver, PhD Thesis, Oxford University, 1999.

\section{APPENDIX}

\section{Stiffness matrix}

In the moving mesh method introduced, material properties have to be assigned to the mesh through the specification of the local stiffness matrix $\left[\mathbf{K}^{H}\right]$. A good choice in the definition of $\left[\mathbf{K}^{H}\right]$ may have a strong impact on the robustness of the method and on the quality of the deformed mesh. A variety of methods exist in the literature, all relying on the idea of introducing a mechanism controlling the deformation of the elements which are close to collapsing.

In the elastic medium analogy, the construction of the stiffness matrix follows the lines of the classic structural analysis. The spatial coordinate vector $\mathbf{r}$, and the displacement vector $\mathbf{u}$ in the element are modelled as linear combinations of shape functions weighted by the nodal values:

$$
\mathbf{u}(x, y, z)=[\mathbf{N}]\left\{\mathbf{u}^{H}\right\} \quad ; \quad \mathbf{r}(x, y, z)=[\mathbf{N}]\left\{\mathbf{r}^{H}\right\}
$$

with $[\mathbf{N}]$ being the $3 \times 24$ matrix of the shape functions. Now, let $\{\epsilon\}=\left\{\epsilon_{x}, \epsilon_{y}, \epsilon_{z}, \gamma_{x y}, \gamma_{x z}, \gamma_{y z}\right\}^{T}$ be the set of strain components. The relation between strain and displacements is expressed as

$$
\{\epsilon\}=[\mathbf{D}] \mathbf{u}=[\mathbf{D}][\mathbf{N}]\left\{\mathbf{u}^{H}\right\}=[\beta]\left\{\mathbf{u}^{H}\right\},
$$

where $[\mathbf{D}]$ is the usual $6 \times 3$ matrix of differential operators that convert displacements to strain according to the linear elasticity theory [20]. On the other hand, in an elastic material the stress components $\{\sigma\}=\left\{\sigma_{x}, \sigma_{y}, \sigma_{z}, \tau_{x y}, \tau_{x z}, \tau_{y z}\right\}^{T}$ are linked to the deformation through the Hooke's law:

$$
\{\sigma\}=[\mathbf{E}]\{\epsilon\},
$$

in which the elastic matrix $[\mathbf{E}]$ is expressed in terms of two parameters only, namely Young's modulus $E$ and the Poisson coefficient $\nu$, characterizing entirely the elastic properties of the material. The energy balance for the element $H$ is written as

$$
\left\{\mathbf{u}^{H}\right\}^{T}\left\{\mathbf{F}^{H}\right\}=\int_{V}\{\epsilon\}^{T}\{\sigma\} d V
$$


with the integral extended to the volume $V$ of the element $H$. Developing the term on the right hand side yields:

$$
\begin{aligned}
\int_{V}\{\epsilon\}^{T}\{\sigma\} d V & =\int_{V}\left([\beta]\left\{\mathbf{u}^{H}\right\}\right)^{T}[\mathbf{E}]\left([\beta]\left\{\mathbf{u}^{H}\right\}\right) d V \\
& =\left\{\mathbf{u}^{H}\right\}^{T}\left[\int_{V}[\beta]^{T}[\mathbf{E}][\beta] d V\right]\left\{\mathbf{u}^{H}\right\} .
\end{aligned}
$$

The stiffness matrix of the element is therefore given by:

$$
\left[\mathbf{K}^{H}\right]=\int_{V}[\beta]^{T}[\mathbf{E}][\beta] d V .
$$

The evaluation of the integral in (28) is made mapping the element $H$ into a standard hexahedral element through the geometric transformation given by the second of (23). The standard element is assumed to be the bi-unit cubic and the shape functions employed are trilinear (linear in each coordinate):

$$
N_{i}(\xi, \eta, \zeta)=\frac{1}{8}\left(1+\xi \xi_{i}\right)\left(1+\eta \eta_{i}\right)\left(1+\zeta \zeta_{i}\right) \quad i=1 \ldots 8
$$

with $(\xi, \eta, \zeta)$ representing the natural coordinate system, and $\left(\xi_{i}, \eta_{i}, \zeta_{i}\right)$ a vertex of the cube. Thus, denoting by $|J|$ the Jacobian associated with the mapping (23), an integral over the physical element is computed using a Gauss-Legendre quadrature formula [21] over the standard hexahedron:

$$
\int_{V} q(\mathbf{x}) d V=\int_{\Omega} q[\mathbf{x}(\xi, \eta, \zeta)]|J| d \xi d \eta d \zeta \simeq \sum_{k=1}^{n} \sum_{j=1}^{n} \sum_{i=1}^{n} w_{i} w_{j} w_{k} q\left(\xi_{i}, \eta_{j}, \zeta_{k}\right)|J| .
$$

The number of points used in the quadrature formula, directly related to the accuracy of the numerical integration [21], is $n^{3}$. Our experiments suggest that a value of $n=2$ is already adequate for this kind of applications.

We now discuss the specification of the elastic matrix $[\mathbf{E}]$. The Young's modulus $E$ represents the stiffness of the material: larger values correspond to stiffer material. Poisson's ratio $\nu$ measures the transversal shortening undergone by the material when subjected to an extension in the axial direction. Some authors choose the Young's modulus to be inversely proportional to the element area [22, 23], rendering stiffer those elements which in a typical CFD mesh are near the boundaries. Hsu and Chang [15] propose two consecutive stress analysis. The first of these, performed with homogeneous elastic properties, is used to determine an appropriate value of $E$ for the second analysis, based on the application of a failure criterion for the material. Poisson's ratio is generally given a uniform value of zero. We have followed the procedure suggested by Stein et al. [14]. First, we drop the Jacobian in the evaluation of the volume integrals by (30). This determines a treatment of the mesh deformation based on the element size. The specification of $E$ and $\nu$ is derived from constraints of different nature. Consider the decomposition of the strain tensor $[\epsilon]$ in its deviatoric and volumetric components:

$$
[\epsilon]=\frac{(\nabla \mathbf{u})+(\nabla \mathbf{u})^{T}}{2}=[\epsilon]^{o}+\frac{(\nabla \cdot \mathbf{u})}{3} \mathbf{I} .
$$


The matrix formulation of the Hooke's law (25) relates stress and strain stress tensor $[\tau]$ as follows:

$$
[\tau]=\lambda(\nabla \cdot \mathbf{u}) \mathbf{I}+2 G[\epsilon]
$$

where $G$ and $\lambda$ are the first and second Lamé constants, functions of $E$ and $\nu$ only:

$$
\lambda=\frac{\nu E}{(1+\nu)(1-2 \nu)} \quad G=\frac{E}{2(1+\nu)} .
$$

Substituting eq. (31) into (32) one has:

$$
[\tau]=(\lambda+2 G / 3)(\nabla \cdot \mathbf{u}) \mathbf{I}+2 G[\epsilon]^{o} .
$$

The last equation states that for an elastic deformation, changes of shape and changes of volume are weighted by $2 G$ and $(\lambda+2 G / 3)$, respectively. If we measure for each element of the original mesh the shape and volume deformations associated with the transformation from the standard cube, and denote by $\left|\phi_{s}\right|$ and $\left|\phi_{v}\right|$ these quantities, one can finally pinpoint $G$ and $\lambda$ by solving the system

$$
\begin{aligned}
2 G & =\alpha_{s}\left|\phi_{s}\right| \\
\lambda+\frac{2 G}{3} & =\alpha_{v}\left|\phi_{v}\right|,
\end{aligned}
$$

in which $\alpha_{s}$ and $\alpha_{v}$ are two user-input parameters, problem dependent, whose calibration serves to bias the deformation as shape oriented or volume oriented. In conditions of equilibrium one has:

$$
\left\|[\epsilon]^{o}\right\| \sim \frac{\alpha_{v}\left|\phi_{v}\right|}{\alpha_{s}\left|\phi_{s}\right|}\|(\nabla \cdot \mathbf{u}) \mathbf{I}\|
$$

According to eq. (36), for a given finite ration $\left|\phi_{v}\right| /\left|\phi_{s}\right|$, when $\alpha_{v} / \alpha_{s} \rightarrow 0$ the shape deformation is expected to be much smaller than the volume deformation. With this choice $\left(\alpha_{v} / \alpha_{s}=0\right)$ the convergence is fast but the quality is poor because oversized elements occur. Starting from this condition, the ratio can gradually be increased to make the grid stiffer to volumetric changes. This also renders the computation more demanding, so that a compromise has to be made between efficiency and mesh quality. In our experience, $\alpha_{v}$ should be maintained some orders of magnitude (1-3) smaller than $\alpha_{s}$, but this may change depending on the value of $\left|\phi_{v}\right| /\left|\phi_{s}\right|$, which is problem dependent. 